\title{
Design of LED Free-form Lens for Sensor Systems
}

\author{
Chi-Feng Lin*, Yu-Bin Fang, and Wei-Chih Su \\ National Center for High-performance Computing, National Applied Research Laboratories, \\ No. 28, Nanke 3rd Rd., Sinshih Dist., 744 Tainan, Taiwan
}

(Received April 12, 2019; accepted May 1, 2020)

Keywords: LED, sensor system, optical lens

Free-form lenses are commonly used for controlling the ray direction of LEDs, particularly when a uniform lighting distribution for sensor applications is required. We propose a target grid modification (TGM) method based on Snell's law, the edge ray principle, and a luminous flux mapping technique to facilitate the design of an LED free-form lens with uniform illuminance properties over a rectangular area. In the proposed method, the size of grids on the target plane is adjusted adaptively in such a way as to improve the uniformity of the illuminance distribution. It is shown that for a given illumination specification, the optimal parameters applied in the TGM method can be easily determined using a pattern search algorithm. The validity of the proposed approach is demonstrated both numerically and experimentally. The results show that the TGM method improves the illuminance uniformity from 50 to $86 \%$ and yields an LED output efficiency of $90 \%$ given a rectangular target plane with dimensions of $2000 \times 750 \mathrm{~mm}^{2}$ and a lens-to-target plane distance of $500 \mathrm{~mm}$.

\section{Introduction}

LEDs are used in many different products, including mobile equipment (e.g., cellular phones and notebook computers), backlight modules, sensor systems, and general lighting systems. ${ }^{(1-3)}$ However, LEDs have highly directional light-emitting properties, and thus the design of effective optical sensor systems poses a significant challenge. For example, designing a uniform emitting backlight system involves the analysis and optimization of a complex system of light guides, lenses, and reflectors, each with its own geometric and optical properties. In this study, we consider the problem of realizing an LED free-form lens capable of providing the rectangular illumination zone required for a sensor system.

Two methods are currently available for the design of free-form lenses. The first method, known as the simultaneous multiple surface (SMS) method, ${ }^{(4)}$ generates two controlled surfaces to define the two edges of the extended light source. This kind of design has been extensively applied in solar concentrators and automobile headlight systems. ${ }^{(5-7)}$ The second method, referred to as "surface tailoring", constructs a surface such that each ray is oriented towards a certain predefined location. ${ }^{(8)}$ Many studies have shown that by applying the surface tailoring

*Corresponding author: e-mail: knif@narlabs.org.tw https://doi.org/10.18494/SAM.2020.2905 
method in conjunction with an energy mapping approach, ${ }^{(9-13)}$ the flux emitted from the source can be redistributed in such a way as to obtain a specific illuminance distribution on the target plane. For simple designs, the source is small enough to be treated as a point source when designing the lens or reflectors. For 2D design problems, the surface tailoring method enables the construction of a highly accurate lens surface. However, in 3D design problems, large errors can occur when constructing free-form lens models. ${ }^{(10,14)}$ Various researchers have proposed resolving this problem by designing the lens as a series of separate zones. ${ }^{(10,11,15)}$ However, the resulting discontinuous surfaces within the lens structure complicate the fabrication process and limit the potential for mass production. For actual LED lens design, the chip size of the LED should be regarded as an extended source with respect to the secondary lens. The illumination pattern is distorted as a result of the difference between the point source considered in the design phase and the extended source used in practice. The combined errors arising from inaccuracies of the lens surface construction and the effects of the extended source can cause the actual illuminance distribution to deviate significantly from the design specification. As a result, there is a requirement for robust design methods capable of optimizing the design of LED free-form lens systems.

To meet this requirement, we propose a target grid modification (TGM) method based on Snell's law, the edge ray principle, and a luminous flux mapping concept, in which the size of grids on the target plane is adjusted adaptively in such a way as to improve the uniformity of the illuminance distribution. The proposed method is used to design an LED free-form lens capable of providing uniform illuminance over a rectangular area. The validity of the proposed method is demonstrated by comparing the illuminance distribution obtained using a prototype free-form lens with that obtained from ray-tracing simulations.

\section{Methodology for Construction of Optical Lens Model}

\subsection{Luminous flux mapping relationship}

In general, the radiation pattern of a light source and the illuminance distribution of the target plane are axially symmetric. As a result, only one quarter of the geometric model needs to be considered in the design process. Assume that the quarter-radiation pattern of the light source is partitioned into $M$ grids in the latitudinal direction and $N$ grids in the longitudinal direction [see Fig. 1(a)]. As shown in Fig. 1(b), the grid pattern of the illuminance distribution on the target plane is the same as that of the light source. Thus, in both the latitudinal and longitudinal directions, the grids of the light source and target plane have the same luminous flux. The flux of the $i$ th grid of the light source in the latitudinal divisions can be expressed as

$$
\Phi_{\theta}(i)=\Phi_{I} / M=\Delta \varphi \int_{\theta_{i-1}}^{\theta_{i}} I(\theta) \sin \theta d \theta, i=1-M,
$$

where $\theta_{i}$ is the rear edge angle of the $i$ th grid with respect to the principal optical axis; $\theta_{i-1}$ is either the front edge angle of the $i$ th grid with respect to the principal optical axis or the rear 


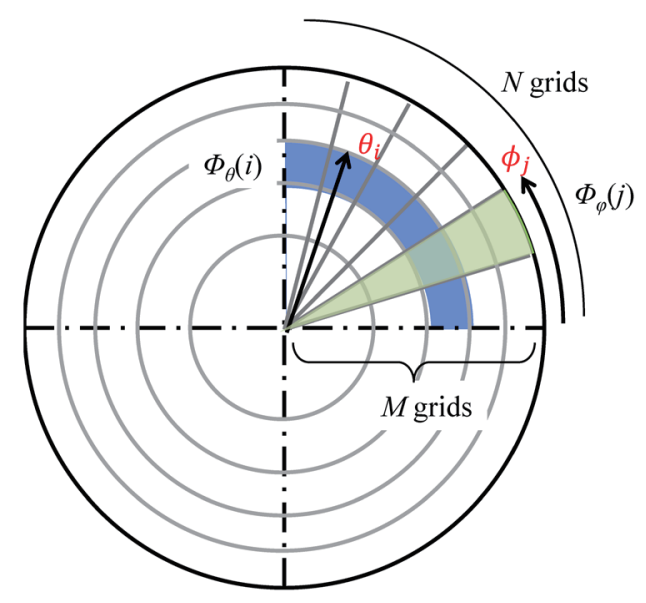

(a)

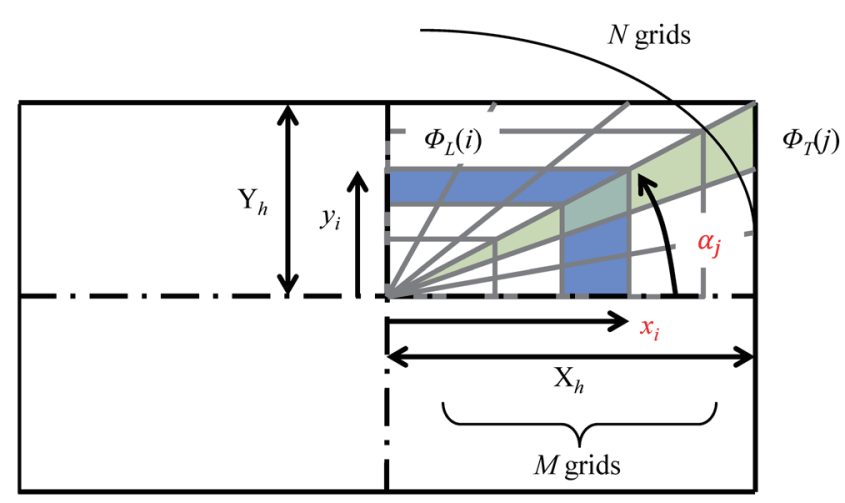

(b)

Fig. 1. (Color online) Schematic illustration showing luminous flux mapping relationship between (a) light source and (b) target plane.

edge angle of the $(i-1)$ th grid; and $\Delta \varphi$ is a fixed value for all of the longitudinal grids. As shown in Eq. (1), the flux $\Phi_{\theta}$ for each grid is obtained by dividing the total flux $\left(\Phi_{I}\right)$ into $M$ parts. Moreover, the angle $\theta_{i}$ of each grid can be obtained by expanding the integration term in Eq. (1).

The flux of the $j$ th grid of the light source in the longitudinal divisions is expressed as

$$
\Phi_{\varphi}(j)=\Phi_{I} / N=\Delta \theta \int_{\varphi_{j-1}}^{\varphi_{j}} I(\varphi) \sin \varphi d \varphi, j=1-N
$$

where $\varphi_{j}$ is the rear edge angle of the $j$ th grid with respect to the axis perpendicular to the principal optical axis [as shown by the $X$-axis in Fig. 1(b)]; $\varphi_{j-1}$ is either the front edge angle of the $j$ th grid or the rear edge angle of the $(j-1)$ th grid; and $\Delta \theta$ is a fixed value for all the latitudinal grids. The flux $\Phi_{\varphi}$ for each longitudinal grid is obtained by dividing the total flux $\left(\Phi_{I}\right)$ into $N$ parts. Moreover, the value of $\varphi_{j}$ for each grid can be obtained by expanding the integration term in Eq. (2).

According to the luminous flux mapping relationship between the light source and the target plane, the L-shaped grids of the target plane in the latitudinal direction [Fig. 1(b)] correspond to the arc-shaped grids of the light source [Fig. 1(a)]. The flux of the $i$ th target grid in the latitudinal divisions can thus be expressed as

$$
\Phi_{L}(i)=\Phi_{O} / M=E \Delta A_{L i}=E\left(A_{L i}-A_{L(i-1)}\right), \text { where } A_{L i}=x_{i} y_{i}, i=1-M,
$$

where $E$ is the average illuminance of each grid on the target plane and $\Delta A_{L i}$ is the grid area obtained by subtracting $A_{L(i-1)}$ from $A_{L i}$, where $A_{L i}$ is the product of the length $x_{i}$ and the width $y_{i}$ of the $i$ th grid. (Note that the ratio of $x_{i}$ to $y_{i}$ is the same as that of the length of the target plane to the width.) As shown in Eq. (3), the flux $\Phi_{L}$ of each grid is obtained by dividing the 
total flux $\left(\Phi_{O}\right)$ of the target plane into $M$ parts. Moreover, the length $x_{i}$ of each grid is calculated using Eq. (3).

In the same way, the triangle-shaped grids of the target plane in the longitudinal direction correspond to the fan-shaped grids of the light source. The flux of the $j$ th target grid following the longitudinal divisions can thus be expressed as

$$
\begin{gathered}
\qquad \Phi_{T}(j)=\Phi_{O} / N=E \Delta A_{T j}=E\left(A_{T j}-A_{T(j-1)}\right), \\
\text { where } A_{T j}= \begin{cases}0.5 X_{h}^{2} \tan \alpha_{j}, & \text { if } A_{T j} \leq 0.5 X_{h} Y_{h}, \quad j=1-N, \\
X_{h} Y_{h}-0.5 Y_{h}^{2} \tan \left(90-\alpha_{j}\right), & \text { if } A_{T j}>0.5 X_{h} Y_{h},\end{cases}
\end{gathered}
$$

where $X_{h}$ is the half-length of the target plane and $Y_{h}$ is the half-width. Moreover, $\Delta A_{T j}$ is the jth grid area obtained by subtracting $A_{T(j-1)}$ from $A_{T j}$, where $A_{T j}$ is the triangular area spanned by the angle $\alpha_{j}$. Note that the angle $\alpha_{j}$ for each grid is obtained by solving Eq. (4).

The edge conditions $\left(\theta_{i}, \varphi_{j}, x_{i}, \alpha_{j}\right)$ for each grid on the light source and target plane can be calculated directly from the luminous flux mapping relationship described above. The edge conditions can then be used to determine all the incident rays and refracted rays for each particular grid. Finally, the points of intersection of the incident rays and refracted rays can be calculated using Snell's law to form the lens surface. The emitting surface of the optical lens model can then be constructed by integrating all of the points of intersection of the incident and refracted rays using computer-aided design (CAD) software. ${ }^{(16)}$

\subsection{Design problem}

The LUXEON Rebel LED produced by Philips Lumileds Ltd. was chosen as the light source for the current design problem. The LED has a light-emitting surface with dimensions of $1.4 \times 1.4 \mathrm{~mm}^{2}$ and radiates light as a Lambertian source. The target plane was assumed to have dimensions of $2000 \times 750 \mathrm{~mm}^{2}$ (length $\times$ width) and was located at a distance of $500 \mathrm{~mm}$ from the LED as shown in Fig. 2. It was assumed that a free-form polymethyl methacrylate (PMMA) optical lens with a refractive index of 1.49 was placed immediately in front of the LED. In developing the optical model of the free-form lens, the quarter-regions of the light source and target plane were both divided into $250(M) \times 250(N)=62500$ grids in accordance with the luminous flux mapping relationship. Figure 3 shows the ray-tracing simulation results obtained for the resulting illuminance of the rectangular target plane. It is seen that the central region of the target plane is illuminated too brightly, while the outer region is illuminated too weakly. Moreover, the illumination of the target plane near the $X$-axis is greater than that near the $Y$-axis. From inspection, the illuminance uniformity (defined as the ratio of the minimum lux to the average lux) is just $50 \%$. The poor uniformity of the illuminance distribution is thought to be the result of two main factors, namely, (1) the surface normal of the lens model constructed using CAD software is different from that calculated from the luminous flux mapping relationship, and (2) the refracted rays emitted by the flat light source deviate from the predetermined rays emitted by the point light source. 


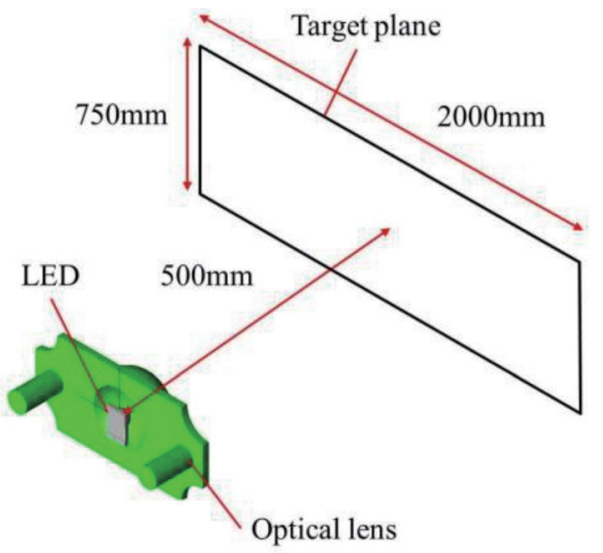

Fig. 2. (Color online) Schematic illustration of LED illumination device and illumination requirement.

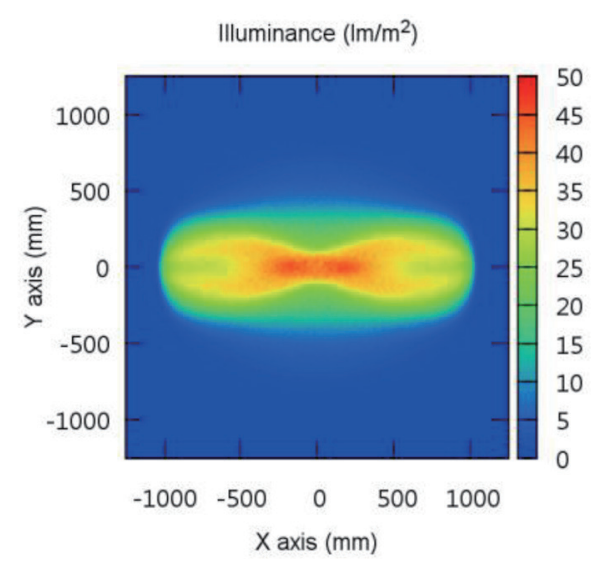

Fig. 3. (Color online) Simulation results showing non-uniform illuminance distribution when TGM method is not applied.

\subsection{TGM method}

We next present a method we call the TGM method for improving the uniformity of the illuminance distribution by adjusting the size of the individual grids on the target plane and reconstructing the optical lens model accordingly. As shown in Fig. 3, the illuminance of the center grids in the latitudinal direction is greater than that of the outer grids. Accordingly, in the proposed TGM method, the illuminance of the central grids is reduced by increasing the grid size on the basis of the luminous flux conservation between the input flux and the output flux over all the grids. Similarly, the illuminance of the edge grids is increased by reducing the grid size subject to the same constraint. In implementing the TGM method, it is assumed that the grid size decreases linearly from the first grid $\Delta A_{L 1}$ in the latitudinal direction to the final grid $\Delta A_{L M}$. Let the ratio of $\Delta A_{L M}$ to $\Delta A_{L 1}$ be denoted as $R A_{L}$. Furthermore, let the value of $R A_{L}$ be bounded by the interval [0.1,1.0], where $R A_{L}=1.0$ indicates that the size of the last grid is equal to that of the first grid. Figures 4(a) and 4(b) show the grid patterns on the target plane corresponding to $R A_{L}=1$ and $R A_{L}=0.5$, respectively. Meanwhile, Fig. 4(c) shows the simulation results obtained for the illumination distribution on the target plane given $R A_{L}=0.5$. Comparing the results presented in Fig. 4(c) with those shown in Fig. $3\left(R A_{L}=1\right)$, it is seen that a reduction in the value of $R A_{L}$ effectively reduces the illuminance of the central grids and increases the illuminance of the outer grids.

As shown in Fig. 3, the illuminance of the grids near the $X$-axis in the longitudinal direction is greater than that of the grids near the $Y$-axis. Accordingly, in the proposed TGM method, the size of the grids near the $X$-axis is increased in order to decease the illuminance, while that of the grids near the $Y$-axis is reduced in order to increase the illuminance. Assume that the grid size decreases linearly from the first grid $\Delta A_{T 1}$ in the longitudinal direction to the final grid $\triangle A_{T N}$. Assume further that the ratio of $\Delta A_{T N}$ to $\Delta A_{T 1}$ is denoted as $R A_{T}$. As with the parameter $R A_{L}$, the value of $R A_{T}$ is bounded by the interval [0.1, 1.0]. Figures 5(a) and 5(b) show the grid patterns on the target plane corresponding to $R A_{T}=1.0$ and $R A_{T}=0.5$, respectively. In addition, 


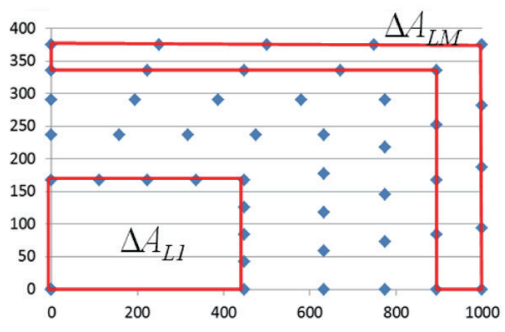

(a)

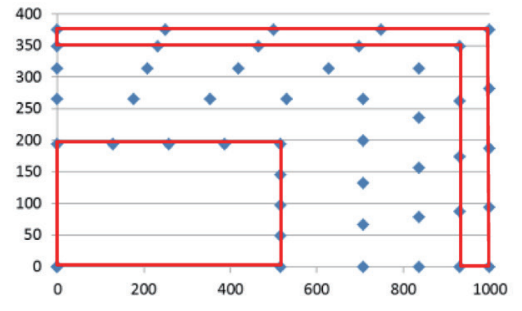

(b)

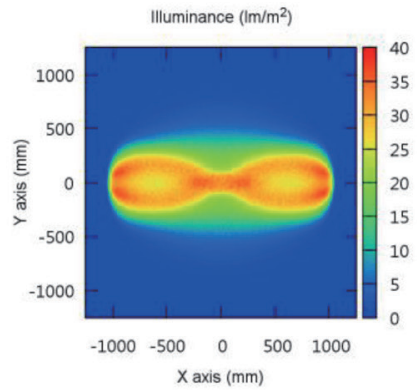

(c)

Fig. 4. (Color online) (a) Target grid pattern with $R A_{L}=1$, (b) target grid pattern with $R A_{L}=0.5$, and (c) illuminance distribution given $R A_{L}=0.5$.

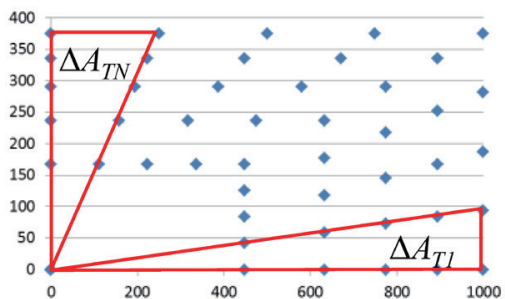

(a)

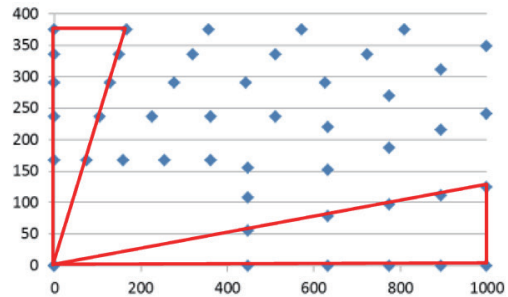

(b)

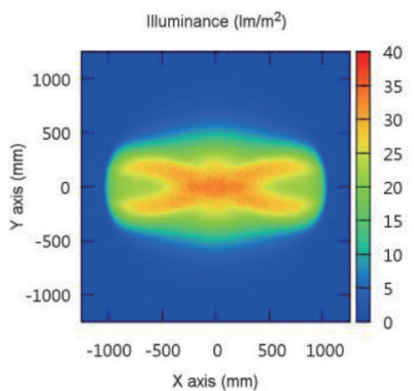

(c)

Fig. 5. (Color online) (a) Target grid pattern with $R A_{T}=1$, (b) target grid pattern with $R A_{T}=0.5$, and (c) illuminance distribution given $R A_{T}=0.5$.

Fig. 5(c) shows the simulated illuminance distribution on the target plane given $R A_{T}=0.5$. By comparing the illuminance distributions shown in Figs. 3 and 5(c), it is seen that a lower value of $R A_{T}$ reduces the illuminance of the grids near the $X$-axis and increases that of the grids near the $Y$-axis.

\section{Simulation and Experimental Results}

Through the simultaneous adjustment of both TGM parameters (i.e., $R A_{L}$ and $R A_{T}$ ), it is possible to adjust the illuminance of each grid on the target plane in both the longitudinal and latitudinal directions. Since the two parameters are limited to values in the range of $0.1-1.0$, it is not difficult for an optimization program to search for the best values of these parameters. To find the uniform illumination, a pattern search algorithm, which is a non-gradient-based method, was used to optimize the design. This optimization method does not need users to provide a detailed mathematical model to evaluate the merit function. Rather, the search algorithm uses the outputs from several ray-tracing simulations as the basis for estimating the possible local minimum and then suggests modified input parameters for the following simulation run. ${ }^{(17)}$ 
Figure 6 presents the simulation results obtained for the illuminance distribution on the target plane given the optimal values of the two TGM parameters, namely, $R A_{L}=0.6$ and $R A_{T}=0.6$. From inspection, the illuminance uniformity is found to be $86 \%$. By contrast, that of the original illuminance distribution [Fig. 3] is only 50\%. In other words, the TGM method significantly improves the illuminance uniformity of the LED free-form lens. Note that the light output efficiency is equal to around $90 \%$ in both cases.

Figures 7(a)-7(c) respectively show the optical lens model, lens prototype, and LED illumination device constructed in accordance with the simulation results presented in Fig. 6. Figure 8 shows the experimental setup used to verify the performance of the LED illumination device. Note that the lens-to-target plane distance was set in accordance with the dimension shown in Fig. 2 (i.e., $500 \mathrm{~mm}$ ). Figure 9 shows the experimental and simulation results obtained for the normalized illuminance intensity curves in the horizontal and vertical directions. It is seen that good qualitative agreement exists between the two sets of results. In general, the results confirm that the TGM-designed LED free-form lens provides a uniform illumination distribution over the specified rectangular area and is therefore suitable for sensor system applications.

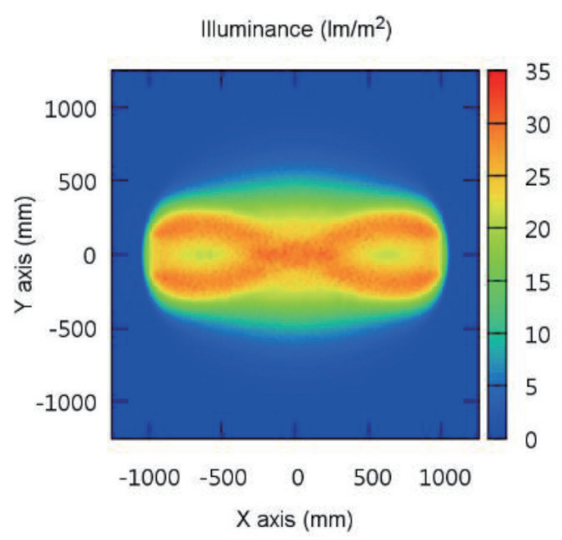

Fig. 6. (Color online) Simulation results showing uniform illuminance distribution when TGM method is applied with $R A_{L}=0.6$ and $R A_{T}=0.6$.

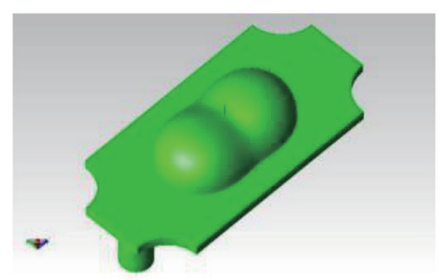

(a)

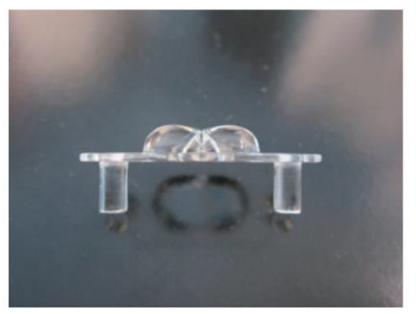

(b)

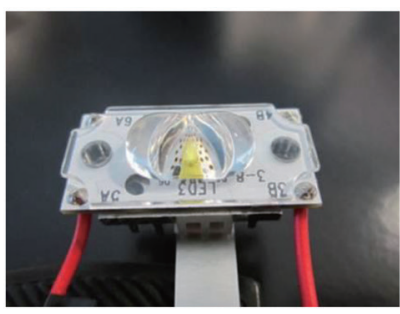

(c)

Fig. 7. (Color online) (a) Computer-generated model of optical lens, (b) prototype of TGM-designed LED freeform lens, and (c) LED illumination device. 


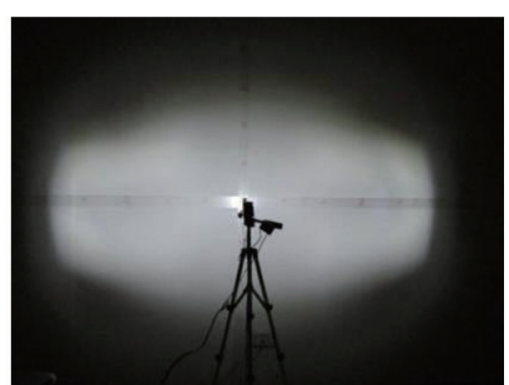

Fig. 8. Photograph showing experimental setup of LED illumination device.

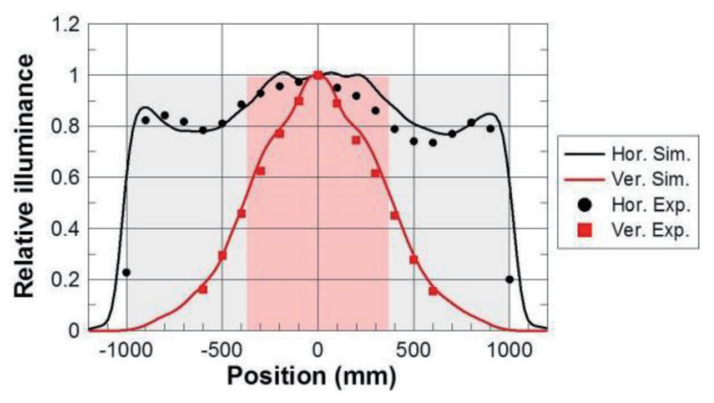

Fig. 9. (Color online) Experimental and simulation results obtained for illuminance distribution of LED illumination device.

\section{Conclusions}

We proposed a TGM method based on a luminous flux mapping technique for optimizing the design of an LED free-form lens for sensor systems. In the proposed approach, the size of the individual grids on the target plane is adjusted adaptively in such a way as to achieve a uniform illuminance distribution. Importantly, the optimal values of the two grid-size adjustment parameters in the TGM method can be easily determined using a simple pattern search algorithm. The simulation results have shown that when the proposed TGM method is not applied, the illuminance uniformity has a value of just 50\%. However, when the TGM method is applied, the illuminance uniformity increases to around $86 \%$. The experimental results confirmed the simulation findings and showed that the TGM-designed LED free-form lens is suitable for sensor systems.

\section{Acknowledgments}

We would like to express our sincere gratitude for the support from the Ministry of Science and Technology of the Republic of China, Taiwan, under Contract Nos. MOST 108-2622-8-006014 and MOST 108-2221-E-492-021-MY2.

\section{References}

D. G. Pelka and K. Patel: Proc. SPIE 5186 Design of Efficient Illumination Systems (2003) 15-26.

2 I. Gerhardt, L. Mai, A. Lamas-Linares, and C. Kurtsiefer: Sensors 11 (2011) 905.

K. Wang and S. Liu: Proc. 8th Int. Conf. Electronic Packaging Technology (IEEE, 2007) 1.

4 J. C. Miñano and J. C. González: Appl. Opt. 31 (1992) 3051.

5 J. C. Miñano, P. Benítez, and J. C. González: Appl. Opt. 34 (1995) 2226.

6 J. C. Miñano, J. C. González, and P. Benítez: Appl. Opt. 34 (1995) 7850.

7 A. Cvetkovic, O. Dross, J. Chaves, P. Benitez, J. C. Miñano, and R. Mohedano: Opt. Express 14 (2006) 13014.

8 H. R. Ries and R. Winston: J. Opt. Soc. Am. A 11 (1994) 1260.

9 W. B. Elmer: The Optical Design of Reflectors (Wiley, New York, 1980).

10 K. Wang, S. Liu, F. Chen, Z. Qin, Z. Liu, and X. Luo: J. Opt. A: Pure Appl. Opt. 11 (2009) 105501.

11 L. Wang, K. Qian, and Y. Luo: Appl. Opt. 46 (2007) 3716.

12 P. Goldstein: Opt. Eng. 50 (2011) 121703. 
13 Y. Ding, X. Liu, Z. Zhang, and P. Gu: Opt. Express 16 (2008) 12958.

14 F. R. Fournier, W. J. Cassarly, and J. P. Rolland: Opt. Express 18 (2010) 5295.

15 W. A. Parkyn: Proc. SPIE 3779 Current Developments in Optical Design and Optical Engineering VIII (1999) 363-370.

16 K. Wang, F. Chen, Z. Liu, X. Luo, and S. Liu: Opt. Express 18 (2010) 413.

17 C. T. Kelley: Iterative Methods for Optimization (SIAM, Philadelphia, 1999).

\section{About the Authors}

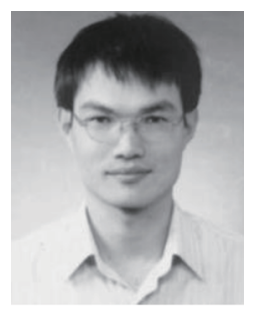

Chi-Feng Lin received his M.S. and Ph.D. degrees in mechanical engineering from National Cheng Kung University, Taiwan, in 1996 and 2002, respectively. He is currently an associate researcher with the National Center for High-performance Computing, Taiwan. His research focuses on backlight design, sensor systems, and color science.

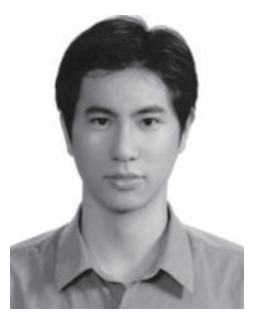

Yu-Bin Fang received his M.S. degree in engineering science from National Cheng-Kung University, Taiwan, in 2004. He is currently an assistant researcher with the National Center for High-performance Computing, Taiwan. His major research interests are the diffuser dot design of light guides for backlight systems and the design of optical illumination systems.

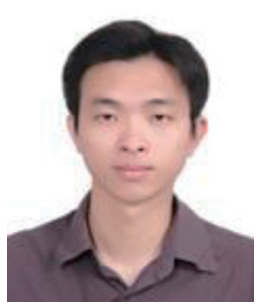

Wei-Chih Su received his Ph.D. degree from the Department of Civil Engineering, National Chiao Tung University, Hsinchu, Taiwan, in 2008. Now, he works at the National Center for High-performance Computing, National Applied Research Laboratories. His current research interests include earthquake signal processing, measurement and analysis of structural dynamic responses, structural health monitoring, and damage assessment. 